\title{
2014 awards in the Journal of Plant Research
}

\author{
Ikuo Nishida
}

Published online: 6 August 2014

(C) The Botanical Society of Japan and Springer Japan 2014

Each year, the Botanical Society of Japan (BSJ) honors excellence in publications of the Journal of Plant Research (JPR) through the Best Paper Awards and the Most-Cited Paper Award. We are proud to announce this year's recipients.

\section{Best Paper Awards}

Munenori Kitagawa and Tomomichi Fujita at Hokkaido University have conducted an imaging study of intercellular protein passage through plasmodesmata in Physcomitrella patens (Kitagawa and Fujita 2013). In plants, plasmodesmata play an important role in cell-to-cell transport of molecules, which must be properly regulated during growth and development. However, it remains unclear when and how the passages of molecules through plasmodesmata are regulated in plants. Using a photoconvertible fluorescent protein, Dendra2, Kitagawa and Fujita have succeeded in demonstrating that a directional passage of some macromolecules through plasmodesmata occurs in protonemata of Physcomitrella patens. They proposed that the directional passages of some macromolecules through plasmodesmata could be affected by photosynthetic and metabolic activities of the cells.

Tomoko Igawa and Yuki Yanagawa at the Nara Institute of Science and Technology and Shin-ya Miyagishima and Toshiyuki Mori at the RIKEN Advanced Science Institute have succeeded in visualizing the membrane dynamics of

I. Nishida $(\bowtie)$

Division of Life Science, Graduate School of Science and

Engineering, Saitama University, 255 Shimo-Okubo, Sakura-Ku,

Saitama 338-8570, Japan

e-mail: jpreic@gr.saitama-u.ac.jp fusing gamete cells during double fertilization of Arabidopsis. Double fertilization, a unique sexual reproduction process in Angiosperms, has been studied for many years by histological observation of non-living cells under an electron microscope, so that our images of double fertilization would be rather static such that one sperm cell fertilizes the egg and another sperm cell fertilizes the central cell. However, for better understanding of the phenomenon, it is essential to use living cells and obtain a live image of subcellular membrane dynamics within gamete cells during double fertilization. To unravel the subcellular mystery of double fertilization, Igawa et al. (2013) have constructed Arabidopsis cell lines, in which the female and male gamete cells are selectively designed to express a fluorescently labeled PIP2a and GCS1-GFP, respectively. They showed that the double fertilization is initiated by plasma membrane fusion between male and female gametes, followed by the entry of sperm internal membrane components into the female gametes, and results in plasmogamy.

\section{Most-Cited Paper Award}

Yasunari Fujita at the Japan International Research Center for Agricultural Sciences (JIRCAS), Miki Fujita and Kazuo Shinozaki at the RIKEN Yokohama Institute, and Kazuko Yamaguchi-Shinozaki at The University of Tokyo have contributed an excellent review article (Fujita et al. 2011) to a JPR symposium entitled "Opening a New Era of ABA Research" (Nambara and Kuchitsu 2011). ABA or abscisic acid plays a pivotal role in a variety of developmental processes. In particular, the role of ABA in cellular dehydration responses, such as during seed maturation and vegetative growth stages, have been extensively studied. 
The review article summarizes recent studies on the ABAmediated transcriptional regulation in response to osmotic stress during vegetative growth stages of Arabidopsis and provides an insightful perspective to future research by leading scientists of this field.

\section{Our new mission}

A new mission has been launched, under the support of the Japan Society for the Promotion of Science (No. 261014), to increase the global credibility of JPR as an international journal of basic plant sciences. The credibility means, to my view, that JPR could continue to publish excellent papers ever more. This year is the first year of our challenge; we will be inviting excellent papers to JPR by strategically encouraging the organization of an international JPR symposium at the BSJ annual meeting.

\section{From the editorial office}

We have recently revised our "Instructions to Authors" (23 July 2014), which include some important downloadable materials, such as a Word template (zip, $154 \mathrm{kB}$ ), "Abbreviations that can be used without definition" (pdf, 7kB), "Typographical Rules" (pdf, 12 kB), "EndNote Style" (zip, $2 \mathrm{kB}$ ), and "Artwork and Illustrations Guidelines" (pdf, $158 \mathrm{kB}$ ).

To maximize discoverability of our articles by indexing services or search engines, the Abstract is becoming more important than ever as a free information source. Thus, we have decided to increase the maximum size of the Abstract from 200 to 300 words.

There seemed to be slight confusion among authors about differences in abbreviation rules recommended by JPR and Springer. We have decided therefore to adopt
Springer's standard abbreviation rules. The major changes are: (1) in the Abstract and in the main text, abbreviations should be spelled out at their first occurrence, with the abbreviations appearing in parentheses; subsequent occurrences should show only the abbreviations; do not use an abbreviation in the Abstract if it appears only once; (2) in tables, abbreviations and their expansions should be given in footnotes; and (3) in figures, abbreviations and their expansions should be given in figure legends (abbreviations in italics and the expansions in roman).

We have been working with a new assistant, Junko Kadkhodaei, in the JPR office since 1 January. Yuko Aoshima, who had dedicated herself to almost all the work in the JPR office since January 2009, had left our office. On behalf of all editors, editorial board members, and authors of JPR, I would like to express my greatest gratitude for her long period of work in the JPR office. We also have been working with a new assistant, Miles Peterson, for assistance in text and proofreading since 1 July.

Ikuo Nishida

Editor-in-Chief, Journal of Plant Research

\section{References}

Fujita Y, Fujita M, Shinozaki K, Yamaguchi-Shinozaki K (2011) ABA-mediated transcriptional regulation in response to osmotic stress in plants. J Plant Res 124:509-525

Igawa T, Yanagawa Y, Miyagishima S, Mori T (2013) Analysis of gamete membrane dynamics during double fertilization of Arabidopsis. J Plant Res 126:387-394

Kitagawa M, Fujita T (2013) Quantitative imaging of directional transport through plasmodesmata in moss protonemata via single-cell photoconversion of Dendra2. J Plant Res 126:577585

Nambara E, Kuchitsu K (2011) Opening a new era of ABA research. J Plant Res 124:431-435 